\title{
The hypernuclear Auger effect within the density dependent relativistic hadron field theory
}

\author{
Christoph Keil and Horst Lenske \\ Institut für Theoretische Physik, University Gießen \\ Heinrich-Buff-Ring 16 \\ D-35392 Gießen
}

(Dated: October 26, 2018)

\begin{abstract}
The hypernuclear Auger effect, given by the de-excitation of a $\Lambda$ hypernucleus by means of the transition of a $\Lambda$ hyperon from an initial to a lower lying final single particle state in conjunction with neutron emission from the core nucleus is studied in relativistic DDRH field theory. Baryonic interactions are obtained from the Bonn NN potential by Dirac-Brueckner theory and theoretically derived scaling laws for the meson-hyperon vertices. The model is applied to the ${ }_{\Lambda}^{209} \mathrm{~Pb}$ hypernucleus. $\Lambda$ and nucleon bound states as well as scattering states are calculated self-consistently in mean-field approximation. The Auger spectra of the emitted neutrons are of complex structure due to a huge combinatorial number of possible hypernuclear transitions. The sensitivity of the neutron Auger spectra to changes in the $\Lambda \sigma$ and $\Lambda \omega$ vertices is investigated. The theoretical results show that experimental applications of the hypernuclear Auger effect will require special efforts, e.g. by tagging on the energy of the initially created hyperon.
\end{abstract}

PACS numbers: $21.80 .+\mathrm{a}$

Keywords: hypernuclei, spectroscopy, Auger

\section{INTRODUCTION}

In order to obtain a more profound understanding of the interactions between baryons with and without strangeness the major goal of hypernuclear physics is to explore the spectral properties of $\Lambda$ hyperons in a nucleus. The experimentally well confirmed existence of $\Lambda$ single particle states [1, 2, 3, 4, 5, 6] indicates the importance of static mean-field interactions. While the overall features are reasonably well reproduced by non-relativistic [7, 8] and relativistic approaches 19, 10, 11 open questions exist on finer details of hypernuclear spectra. In light nuclei, $A \leq 16$, the smallness of the spin-orbit splitting and other fine structures of $\Lambda$ single particle spectra are well described by multi-configuration shell model calculations with empirical matrix elements 12 . These calculations emphasize the importance of residual interactions and configuration mixing. Theoretical studies of dynamical self-energies in $\Lambda$ nuclei, predicting rather strong contributions from the coupling of the $\Lambda$ to excitations of the nuclear core, point in a similar direction [7, 8]. This, however, is not fully complying with recent observations of well separated and relatively sharp $\Lambda$ single particle structures in the medium-mass ${ }_{\Lambda}^{51} \mathrm{~V}$ and ${ }_{\Lambda}^{89} \mathrm{Y}$ hypernuclei [1] [2]. The measured spectra are compatible with a much stronger spin-orbit strength than expected from the low mass region, amounting to spin-orbit interaction energies of 1 to $2 \mathrm{MeV}$, but the data do not indicate a strong damping of the $\Lambda$ single particle states.

The conclusion to be drawn from these - partly conflicting - findings is that there is a clear necessity for more precise measurements and an enlarged body of data. For more decisive results on $\Lambda$-nucleus interactions medium and heavy mass nuclei can be expected to be much better suited. In light nuclei threshold effects from the weak binding of $\Lambda$ states and finite size effects contribute strongly to the observed spin-orbit splitting, thus not giving direct access to the wanted information on the genuine $\Lambda N$ interaction strengths [13]. The calculations in ref. 14 indicate that these effects decrease rapidly beyond the silicon mass region. A standard tool for spectral investigations is $\gamma$-spectroscopy, detecting the photons from the hypernuclear transitions of the $\Lambda$ particle after it was created in an excited state e.g. in a $A(\pi, K)_{\Lambda} A$ reaction. In a recent $\left(K^{-}, \pi^{-}\right)$experiment on ${ }^{13} \mathrm{C}$ at BNL/AGS progress has been made by achieving a much better energy resolution than before [15. These measurements, by the way, confirm the small $\Lambda$ spin-orbit splitting in light nuclei. By technical reasons $\gamma$-spectroscopy is not applicable in medium and heavy mass nuclei.

A promising alternative for spectroscopy in medium and heavy mass hypernuclei is the observation of Auger neutrons, emitted during the de-excitation of the hypernucleus after the initial creation of a $\Lambda$ in an excited state. The hypernuclear Auger process was discussed first by Likar et al. 16. More recently, the idea has been revived and worked out in much more detail for an experiment proposal at JLAB [17]. The hypernuclear Auger effect is the direct de-excitation of an excited $\Lambda$ single-particle state in a hypernucleus by the emission of a neutron. The process is analogous to the well known atomic Auger effect where the de-excitation of an electron kicks out another less bound one. In the case of the hypernuclear Auger effect the energy spectrum of the emitted neutrons reflects the $\Lambda$ singeparticle level structure, but folded with the neutron single-particle spectrum. The mechanism of the hypernuclear Auger effect is schematically shown in figure 1. In order to occur the separation energy of at least the $\Lambda$ 1s-orbit must be larger than the separation energy of the valence neutron. Therefore, because of the much weaker $\Lambda$ binding, the 


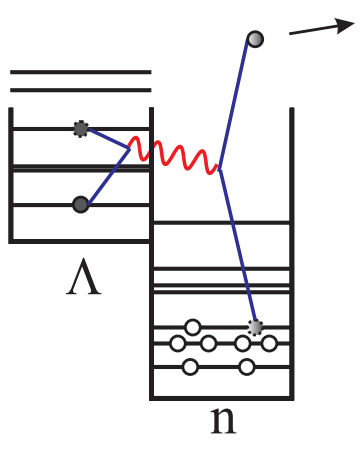

FIG. 1: Schematic picture of the hypernuclear Auger effect: A $\Lambda$ hyperon is de-excited into a lower single particle level,thereby transferring energy and momentum to a valence neutron which is emitted through this process.

hypernuclear Auger effect appears with a strength sufficient for measurements only in intermediate and heavy mass nuclei where neutron and $\Lambda$ separation energies of comparable magnitude are available. Already from this simplified picture it is clear that a single transition of the $\Lambda$ will yield a wealth of peaks in the neutron's energy distribution, requiring a very careful analysis. For the proposed JLAB experiment [17] a neutron energy resolution of better than $50 \mathrm{keV}$ is envisaged. As nuclei to be studied in the experiment $P b$ and $U$ are proposed. In this mass region it can yield valuable information about the $\Lambda$ single particle spectra, possibly even resolving finer details as the spin-orbit splitting. One purpose of this paper is to investigate in a realistic model predictions for spectral distributions which may be used to estimate constraints on future measurements.

In this paper we report on our calculations of the hypernuclear Auger spectra within the density dependent relativistic hadron field theory (DDRH). In section II we describe the theoretical description of the hypernuclear Auger effect in the relativistic DDRH approach with density dependent meson-baryon vertices. We shortly sketch the main features of DDRH and the numerical realization. In section III results are presented and possibilities to extract information from the extremely complex spectra are discussed. In section IV we summarize, discuss our results and draw some conclusions.

\section{THE HYPERNUCLEAR AUGER EFFECT}

\section{A. Formalism}

The emission of a neutron by the $\Lambda$ de-excitation of the hypernucleus is described as a decay of an initial $\Lambda$ single particle state into a 2 particle- 1 hole configuration where the $\Lambda$ is coupled to a neutron particle-hole core excitation with an energy above the particle emission threshold. The nucleon and hyperon single particle states are obtained in relativistic mean-field ( $\mathrm{RMF}$ ) approximation. Residual interactions among the neutron particle-hole configurations are neglected, i.e. an uncorrelated quasiparticle description is used.

For the transition operator $V$ the one-boson-exchange parametrization of the full Dirac-Brueckner (DB) G-matrix with density dependent vertex functionals $\Gamma(\hat{\rho})$ 20 is used. It is the same interaction as applied in the structure calculation. Since the $\Lambda$ hyperon is electrically neutral and an iso-scalar particle only the $\sigma$ and the $\omega$ mesons contribute:

$$
V=\Gamma_{\sigma \Lambda}\left(\hat{\rho}_{\Lambda}\right) \frac{1}{q^{2}-m_{\sigma}^{2}} \Gamma_{\sigma N}\left(\hat{\rho}_{N}\right)-\Gamma_{\omega \Lambda}\left(\hat{\rho}_{\Lambda}\right) \frac{1}{q^{2}-m_{\omega}^{2}} \Gamma_{\omega N}\left(\hat{\rho}_{N}\right)
$$

Taking $|0\rangle$ to be our many-body ground state, assumed here as the $0^{+}$ground state of a spherical nucleus, the initial state is in second quantization formulation given by $a_{\Lambda_{\alpha}}^{\dagger}|0\rangle$, where $a_{\Lambda_{\alpha}}^{\dagger}$ is the creation operator for a $\Lambda$ state with the set of quantum numbers $\Lambda_{\alpha}$. In the final state the hyperon is attached to a particle-hole excited nuclear core. The excess energy and momentum is carried away by the emitted neutron occupying an unbound single particle continuum state in the nuclear mean-field potential:

$$
\left[a_{\Lambda_{\beta}}^{\dagger} \otimes A_{n_{\beta}}^{\dagger}\left(j_{n_{\beta}} j_{n_{\beta}^{-1}}\right)\right]_{j_{\beta} m_{\beta}}|0\rangle=\sum_{m_{\Lambda_{\beta}} M_{n_{\beta}}}\left\langle j_{\Lambda_{\beta}} m_{\Lambda_{\beta}} J_{n_{\beta}} M_{n_{\beta}} \mid j_{\beta} m_{\beta}\right\rangle a_{\Lambda_{\beta}}^{\dagger} A_{n_{\beta}}^{\dagger}\left(j_{n_{\beta}} j_{n_{\beta}^{-1}}\right)|0\rangle
$$


$A_{n_{\beta}}^{\dagger}$ is the particle-hole excitation operator with angular momentum $J_{n_{\beta}}, M_{n_{\beta}}$ defined through:

$$
A_{n}^{\dagger}\left(j, j^{\prime}\right)=\sum_{m, m^{\prime}}\left\langle j m j^{\prime} m^{\prime} \mid J_{n} M_{n}\right\rangle a_{j m}^{\dagger} \tilde{a}_{j^{\prime} m^{\prime}},
$$

where $\tilde{a}=(-)^{j+m} a_{j,-m}$ denotes a hole creation operator. In eq.2 the $a_{\Lambda_{\beta}}^{\dagger}$ and $A_{n_{\beta}}^{\dagger}$ are coupled to total angular momentum $j_{\beta}, m_{\beta}$.

The differential widths $d \Gamma_{j_{\Lambda_{\alpha}}}$ describing the decay of an initial $\Lambda_{\alpha}$ state is determined by the transition matrix elements of the $\Lambda N$ interaction $V$ :

$$
d \Gamma_{j_{\Lambda_{\alpha}}}=\frac{1}{32 \pi^{2}} \frac{1}{2 j_{\Lambda_{\alpha}}+1} \sum_{\left\{\gamma_{1}\right\}}\left|\left\langle 0\left|\left[a_{\Lambda_{\beta}} \otimes A_{n_{\beta}}\left(j_{n_{\beta}}, j_{n_{\beta}^{-1}}\right)\right]_{j_{\beta} m_{\beta}} V a_{\Lambda_{\alpha}}^{\dagger}\right| 0\right\rangle\right|^{2} \frac{|\vec{k}|}{M^{2}} d \Omega
$$

where $\quad\left\{\gamma_{1}\right\}=\left\{m_{\Lambda_{\alpha}}, m_{\beta}, j_{\beta}, j_{\Lambda_{\beta}}, j_{n_{\beta}}, j_{n_{\beta}^{-1}}\right\}$ indicates the incoherent summation over degenerate initial and final sub-states, including the appropriate phase space factors [18] due to the neutron emerging with momentum $\vec{k}$. $M$ is the mass of the initial hypernucleus. The orthogonality and completeness relations of the Clebsch-Gordan coefficients 19] allow to convert this expression into the equivalent form of an incoherent sum over matrix elements of uncoupled states:

$$
d \Gamma_{j_{\Lambda_{\alpha}}}=\frac{1}{32 \pi^{2}} \frac{1}{2 j_{\Lambda_{\alpha}}+1} \sum_{\left\{\gamma_{2}\right\}}\left|\left\langle 0\left|a_{\Lambda_{\beta}} a_{n_{\beta}} \tilde{a}_{n_{\beta}^{-1}}^{\dagger} V a_{\Lambda_{\alpha}}^{\dagger}\right| 0\right\rangle\right|^{2} \frac{|\vec{k}|}{M^{2}} d \Omega
$$

where $\left\{\gamma_{2}\right\}=\left\{m_{\Lambda_{\alpha}}, m_{\Lambda_{\beta}}, m_{n_{\beta}}, m_{n_{\beta}^{-1}}, j_{\Lambda_{\beta}}, j_{n_{\beta}}, j_{n_{\beta}^{-1}}\right\}$. From equations (14) and (5) it is seen that the Auger process is determined by nucleonic particle-hole fluctuations of the nuclear mean-field absorbing the energy-momentum transfer from the $\Lambda$ transition. This structure becomes even more obvious by expressing the matrix elements in terms of the appropriate non-diagonal elements of $\Lambda$ and nucleon one-body density matrices in momentum space:

$$
\left\langle 0\left|a_{\Lambda_{\beta}} a_{n_{\beta}} V a_{n_{\beta}^{-1}}^{\dagger} a_{\Lambda_{\alpha}}^{\dagger}\right| 0\right\rangle=\int d^{4} q \rho_{\Lambda^{\prime} \Lambda}(q) V(q) \rho_{n^{-1} n}(q)
$$

Here, the transition densities $\rho_{i j}$ are given by:

$$
\begin{aligned}
\rho_{i j}(q) & \equiv \int d^{4} x e^{i q x} \bar{\psi}_{i}(x) \hat{\Gamma} \psi_{j}(x) \\
& =\delta\left(\left(q^{0}-\left(E_{i}-E_{j}\right)\right) \int d \Omega d r r^{2}\left(\sum_{\mu, \lambda}(-)^{\lambda} Y_{\lambda \mu}^{*}(\hat{q}) Y_{\lambda \mu}(\hat{r}) j_{\lambda}(q r)\right) \bar{\psi}_{i}(r, \hat{r}) \hat{\Gamma} \psi_{j}(r, \hat{r})\right.
\end{aligned}
$$

$\hat{\Gamma}$ is either $\hat{1}$ or $\gamma^{\mu}$ for $\sigma$ - and $\omega$-exchange, respectively. $E_{i, j}$ are the single particle energies of the states $\psi_{i, j}$. In the second line the spatial part of the plane wave is expanded into partial waves. A more detailed description of the evaluation of the matrix elements is given in appendix A. The wave functions and single particle energies $E_{i, j}$ are taken from a DDRH calculation which is described in section II B.

\section{B. The DDRH Model}

The density dependent relativistic hadron field theory (DDRH) 20, 21] is a relativistic Lagrangian field theory with baryons and mesons interacting by density dependent coupling functionals. The meson-baryon interaction part of the DDRH-Lagrangian used here is chosen as in 20]:

$$
\begin{aligned}
\mathcal{L}_{i n t}= & \bar{\Psi} \Gamma_{\sigma}(\hat{\rho}) \Psi \sigma-\bar{\Psi} \Gamma_{\omega}(\hat{\rho}) \gamma_{\mu} \Psi \omega^{\mu} \\
& -\frac{1}{2} \bar{\Psi} \vec{\Gamma}_{\rho} \gamma_{\mu} \Psi \vec{\rho}^{\mu}-e \bar{\Psi}_{F} \hat{Q} \gamma_{\mu} \Psi_{F} A^{\mu}
\end{aligned}
$$

including isoscalar interactions from the scalar $\sigma$ and the vector $\omega$ meson and isovector vector interactions given by the $\rho$-meson. The last term accounts for the electromagnetic interaction where $\hat{Q}$ is the charge operator. The density 
dependence of the baryon-meson vertices is determined self-consistently in mean-field approximation from DiracBrueckner $(D B)$ self-energies by first solving the Bethe-Salpeter equation for the in-medium two-particle scattering amplitude and then extracting the medium modified vertices by expanding the DB Hartree-Fock self-energies in terms of the meson-exchange self-energies obtained from equation (8) [20, 22]. Medium modifications contribute at various levels, e.g. the dressing of the baryon propagators by self-energies and the Pauli-blocking of intermediate states. A Lorentz invariant and thermodynamically consistent field theory is retained by expressing the density dependent vertices in terms of functionals of Lorentz scalar combinations $\hat{\rho}_{i}$ of the baryon field operators $\psi$ [20]. The description of infinite nuclear matter and pure isospin nuclei within DDRH theory has been shown to be very good using the Bonn A and the Groningen NN-potentials [20, 21].

In 14 the extension of the DDRH theory to the strangeness sector and applications to hypernuclear structure calculations were discussed. Since DB calculations describing the in-medium interactions of the complete baryon octet are not available we follow here the approximation scheme discussed also in [14]. The essential step is obtained from the diagrammatic analysis of DB hyperon self-energies, showing that the coupling functionals of nucleons and hyperons are related in leading order by a simple scaling law given by the ratio of the free space meson-nucleon and meson-hyperon Born-term coupling constants $g_{N \alpha}$ and $g_{Y \alpha}$, respectively [14:

$$
\Gamma_{Y \alpha}\left(\rho_{Y}\right) \approx \frac{g_{Y \alpha}}{g_{N \alpha}} \Gamma_{N \alpha}\left(\rho_{Y}\right) \equiv R_{Y \alpha} \Gamma_{N \alpha}\left(\rho_{Y}\right) .
$$

The scalar scaling factor $R_{\Lambda \sigma}$ was taken from the Jülich-model $\left(R_{\Lambda \sigma}=0.49\right)$ [23], including explicitly $\pi \pi$ and $K \bar{K}$ dynamical correlations in the $0^{+}$scalar-isoscalar meson channel. The scaling factor for the $\Lambda \Lambda \omega$ vector vertex is obtained phenomenologically from a least-square fit to $\Lambda$ separation energies, resulting in $R_{\Lambda \omega}=0.553$ [14]. However, in ref. 114] it was noticed that the binding energies in the light nuclei below oxygen seem to behave differently from the systematics obtained from $A \geq 40$. The $\Lambda$ separation energies in the light nuclei are described with the same accuracy only if the repulsion from the $\omega$ meson is reduced by about $5 \%$ to $R_{\Lambda \omega}=0.542$. DDRH results for single $\Lambda$ hypernuclei are compared to the presently available set of world data in [14]. An agreement on the percent level is obtained, being at least of the same quality as those of purely phenomenological mean-field models.

\section{RESULTS}

In this work we consider the hypernucleus ${ }_{\Lambda}^{209} \mathrm{~Pb}$ as representative for the heavy mass hypernuclei (it is one of the nuclei that are going to be studied in the JLAB experiment). The wave functions and single particle energies used for the evaluation of the matrix elements are calculated self-consistently by solving the DDRH field equations in relativistic mean-field (RMF) approximation. Details of the numerical approach and the model parameters are given in [14]. For the present application we need information on knocked-out, unbound neutron states. For that purpose the single particle continuum was discretized by enclosing the system in a huge box of size $R=150 \mathrm{fm}$. Since the spacing of the discretrized continuum levels behaves as $\sim \mathcal{O}\left(1 / R^{2}\right)$ the use of such a large quantization volume ensures quasi-continuous energy spectra for the neutron scattering states, allowing to resolve single particle resonances and other continuum structures resulting from the calculations.

The continuum wave functions are calculated in the self-consistently obtained ground state mean-field potentials. Thus, final state interactions are taken into account on the level of static mean-field self-energies. The approach assures orthogonality of bound and unbound wave functions thus avoiding the unphysical non-orthogonality contributions inherent to phenomenological approaches.

Since the previous applications of the DDRH theory show that the experimental $\Lambda$ spectra are reproduced especially accurate in heavy nuclei our approach is well suited for the calculation of the Auger neutron spectra from ${ }_{\Lambda}^{209} \mathrm{~Pb}$. Numerical values of the single particle energies for occupied neutron states and bound $\Lambda$ states are displayed in table II.

Because of the high level density and moderate separation energies heavy hypernuclei are most suitable for the Auger effect. However, at the same time these apparent advantages are, unfortunately, a potential source of problems for experimental work. The huge amount of combinatorial possibilities for transitions, illustrated in table II and indicated in figure 2 for the case of initially populating the $1 g^{\Lambda}$-shell in ${ }_{\Lambda}^{209} \mathrm{~Pb}$, leads to Auger spectra of a rather complicated shape making in many cases an unambiguous identification of transitions and assignment of quantum numbers almost impossible.

The problem is apparent from figure 3 where the complete neutron emission spectrum from the ${ }_{\Lambda}^{209} \mathrm{~Pb}$ hypernucleus is displayed, summed over all energetically open $\Lambda$ levels. Experimentally, spectra of a similar structure have to be expected. In addition, state-dependent weighting factors from the production vertex of the initial $\Lambda$ state will be superimposed. From figure 3 and figure 2 it is obvious that experiments will be confronted with spectra of high complexity. Before observables of physical interest can be accessed the data will have to be analyzed in a more selective 


\begin{tabular}{l|r||l|r}
$\Lambda$ & $\mathrm{E}[\mathrm{MeV}]$ & $\mathrm{n}$ & $\mathrm{E}[\mathrm{MeV}]$ \\
\hline $1 \mathrm{~s}_{1 / 2}$ & -27.16352 & $1 \mathrm{~g}_{9 / 2}$ & -27.67206 \\
$1 \mathrm{p}_{3 / 2}$ & -23.30941 & $1 \mathrm{~g}_{7 / 2}$ & -24.97048 \\
$1 \mathrm{p}_{1 / 2}$ & -23.08789 & $2 \mathrm{~d}_{5 / 2}$ & -21.91610 \\
$1 \mathrm{~d}_{5 / 2}$ & -18.53900 & $2 \mathrm{~d}_{3 / 2}$ & -20.61122 \\
$1 \mathrm{~d}_{3 / 2}$ & -17.98296 & $3 \mathrm{~s}_{1 / 2}$ & -19.86137 \\
$2 \mathrm{~s}_{1 / 2}$ & -15.74262 & $1 \mathrm{~h}_{11 / 2}$ & -18.56635 \\
$1 \mathrm{f}_{7 / 2}$ & -13.15220 & $1 \mathrm{~h}_{9 / 2}$ & -14.83666 \\
$1 \mathrm{f}_{5 / 2}$ & -12.11529 & $2 \mathrm{f}_{7 / 2}$ & -12.70419 \\
$2 \mathrm{p}_{3 / 2}$ & -9.64314 & $2 \mathrm{f}_{5 / 2}$ & -10.93869 \\
$2 \mathrm{p}_{1 / 2}$ & -9.25274 & $3 \mathrm{p}_{1 / 2}$ & -10.32673 \\
$1 \mathrm{~g}_{9 / 2}$ & -7.40907 & $3 \mathrm{p}_{3 / 2}$ & -9.64888 \\
$1 \mathrm{~g}_{7 / 2}$ & -5.80963 & $1 \mathrm{i}_{13 / 2}$ & -9.41419 \\
$2 \mathrm{~d}_{5 / 2}$ & -4.00386 & & \\
$2 \mathrm{~d}_{3 / 2}$ & -3.42605 & & \\
$3 \mathrm{~s}_{1 / 2}$ & -3.09504 & & \\
$1 \mathrm{~h}_{11 / 2}$ & -1.56587 & &
\end{tabular}

TABLE I: DDRH results for $\Lambda$ and neutron single particle energies in ${ }_{\Lambda}^{209} \mathrm{~Pb}$ entering into the Auger-calculations. The standard set of interaction parameters was used [14].

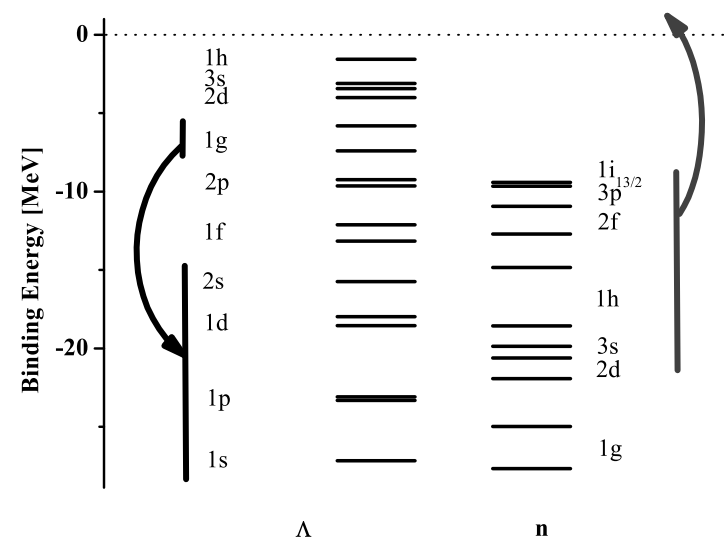

FIG. 2: Level schemes of the $\Lambda$ hyperons and neutrons that are involved in the hypernuclear Auger effect. The levels are displayed in the physical scale as shown in table 1 . The bars mark the single particle levels involved by the de-excitation of an initial $1 \mathrm{~g}$-shell $\Lambda$. The maximum energy, released when the $\Lambda$ drops down to the $1 s$-orbit, allows to emit neutrons from the $1 i_{13 / 2}$ valence orbit down to the $2 d_{3 / 2}$ or $3 s_{1 / 2}$ shells for the $1 g_{7 / 2}$ and $1 g_{9 / 2}$ initial states, respectively.

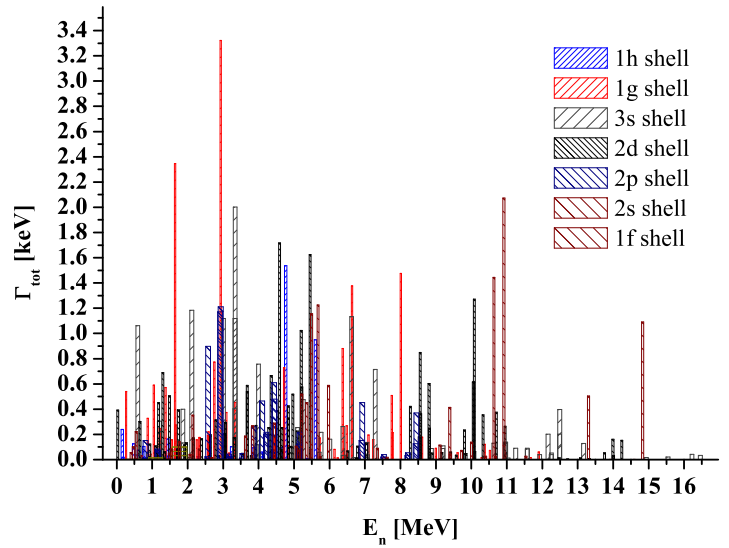

FIG. 3: Full spectrum of the Auger transition strengths in ${ }_{\Lambda}^{209} \mathrm{~Pb}$ including contributions by initial population of the $1 h$, $1 g, 3 s, 2 d, 2 p, 2 s$ and $1 f \Lambda$ orbitals and their subsequent de-excitation by neutron emission.

approach. From the discussion it is obvious that much of the structure will be produced by incoherent superpositions of contributions from the variety of orbitals in which the $\Lambda$ was inititally produced. By a precise energy tagging of the produced kaon and the outgoing electron (for the case of electromagnetic production of hypernuclei, as will be the case at JLAB) it might be possible to determine the initial $\Lambda$-state accurately enough and that only the Auger-neutrons related to the de-excitation of that specific state can be recorded selectively in a coincidence measurement.

In this context, it is of interest to consider the Auger transitions on the limits of the energetically accessible range of states. Assuming energetically sharp states, i.e. the $\Lambda$ and neutrons are in good quasiparticle configurations and damping effects are negligible, the present calculation predicts that the Auger process can only take place if the $\Lambda$ initially is produced in the $2 s^{\Lambda}$-orbit or above (see table $\mathbf{1}$ ), because otherwise the $\Lambda$ transition energies are less than the lowest neutron separation energy. Next to the $2 s^{\Lambda}$-orbit we find the doublet of $1 f^{\Lambda}$ states which is of interest because it allows to observe the $\Lambda$ spin-orbit splitting, at least in principle. Since the energy window available from populating the $1 f^{\Lambda}$-orbits is still rather narrow the resulting Auger neutron spectrum is of a comparatively simple 


\begin{tabular}{|c|c|c|c|}
\hline final $\Lambda$ & neutron-hole & $\| \Gamma_{1 g_{9 / 2}}\left[10^{-2} \mathrm{keV}\right]$ & $\mid \Gamma_{1 g_{7 / 2}}\left[10^{-2} \mathrm{keV}\right]$ \\
\hline \multirow[t]{9}{*}{$1 s_{1 / 2}$} & $2 d_{3 / 2}$ & - & 3.2 \\
\hline & $3 s_{1 / 2}$ & - & 0.6 \\
\hline & $1 h_{11 / 2}$ & 57.1 & 77.3 \\
\hline & $1 h_{9 / 2}$ & 8.7 & 3.5 \\
\hline & $2 f_{7 / 2}$ & 16.0 & 17.8 \\
\hline & $2 f_{5 / 2}$ & 9.1 & 12.2 \\
\hline & $3 p_{3 / 2}$ & 5.5 & 5.5 \\
\hline & $3 p_{1 / 2}$ & 1.8 & 1.7 \\
\hline & $1 i_{13 / 2}$ & 7.4 & 6.1 \\
\hline \multirow[t]{6}{*}{$1 p_{3 / 2}$} & $1 h_{9 / 2}$ & 24.8 & 22.1 \\
\hline & $2 f_{7 / 2}$ & 45.3 & 73.0 \\
\hline & $2 f_{5 / 2}$ & 8.7 & 26.9 \\
\hline & $3 p_{3 / 2}$ & 17.3 & 19.6 \\
\hline & $3 p_{1 / 2}$ & 8.6 & 21.5 \\
\hline & $1 i_{13 / 2}$ & 137.8 & 147.6 \\
\hline \multirow[t]{6}{*}{$1 p_{1 / 2}$} & $1 h_{9 / 2}$ & 4.5 & 17.6 \\
\hline & $2 f_{7 / 2}$ & 37.4 & 17.2 \\
\hline & $2 f_{5 / 2}$ & 11.2 & 1.6 \\
\hline & $3 p_{3 / 2}$ & 8.2 & 5.4 \\
\hline & $3 p_{1 / 2}$ & 8.1 & 2.0 \\
\hline & $1 i_{13 / 2}$ & 88.1 & 51.0 \\
\hline \multirow[t]{4}{*}{$1 d_{5 / 2}$} & $2 f_{5 / 2}$ & 54.1 & 234.6 \\
\hline & $3 p_{3 / 2}$ & 32.9 & 15.7 \\
\hline & $3 p_{1 / 2}$ & 15.9 & 332.2 \\
\hline & $1 i_{13 / 2}$ & 9.4 & 5.3 \\
\hline \multirow[t]{4}{*}{$1 d_{3 / 2}$} & $2 f_{5 / 2}$ & - & 59.0 \\
\hline & $3 p_{3 / 2}$ & $<0.01$ & 27.5 \\
\hline & $3 p_{1 / 2}$ & 0.5 & 17.2 \\
\hline & $1 i_{13 / 2}$ & 1.2 & 19.2 \\
\hline $2 s_{1 / 2}$ & $3 p_{1 / 2}$ & - & $<0.01$ \\
\hline
\end{tabular}

TABLE II: Transitions contributing to the de-excitation of the $1 g^{\Lambda}$-states. Transition widths for the $\Lambda$ initial states $1 g_{9 / 2}$ and $1 g_{7 / 2}$ are denoted by $\Gamma_{1 g_{9 / 2}}$ and $\Gamma_{1 g_{7 / 2}}$, respectively.

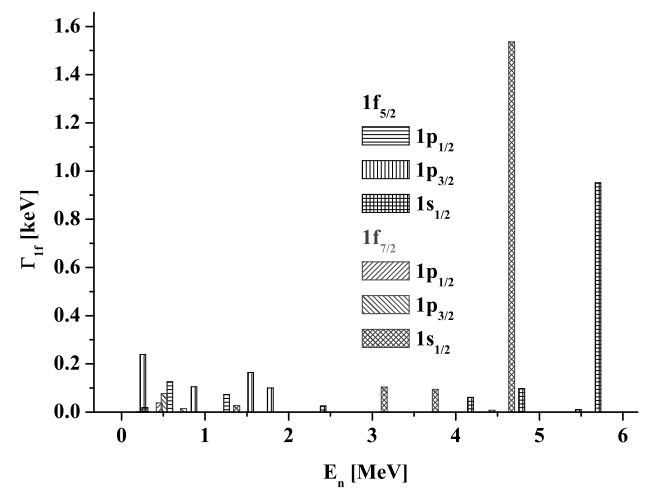

FIG. 4: Decay widths of the Auger transition strengths in ${ }_{\Lambda}^{209} \mathrm{~Pb}$ with an initial $1 \mathrm{f} \Lambda$ state. 
structure. In figure 4 results for the Auger spectra produced by the $1 f_{7 / 2}$ and $1 f_{5 / 2}$ states are compared. It is seen that in both cases only a small number of final states occurs. Even more, only the transitions to the $1 i_{13 / 2}$ neutronhole final state yield a significant strength. Thus it is even possible to clearly obtain the spin-orbit splitting of the $1 f^{\Lambda}$-shell. The prominent strength of this doublet is also fairly model independent. Considering the kinematically allowed phase space of this transition only, one might expect that wave function effects which are sensitive to the details of the interaction could strongly influence the relative strengths between the emission of the $1 i$ - or $3 p$-shell neutrons, which are in energy almost degenerate (see table II). Nevertheless, due to the high degeneracy of the $1 i_{13 / 2}$ neutron-orbitals the multiplicity of these neutrons will be greatly enhanced. Therefore one can expect to observe in the de-excitation spectrum of a $1 f^{\Lambda}$-state a clear line doublet belonging to the two spin-orbit partners of the $1 f^{\Lambda}$ shell falling down to the 1 s orbital and thereby knocking out the $1 i_{13 / 2}$ neutron.

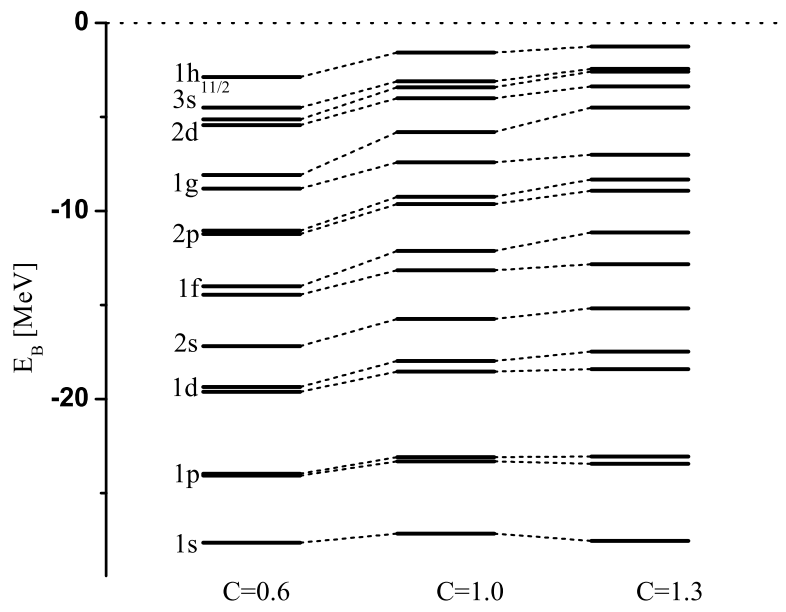

FIG. 5: $\Lambda$ single particle energy spectra in ${ }_{\Lambda}^{209} \mathrm{~Pb}$ for the three different sets of coupling constants discussed in the text.

The general case is, however, more ambiguous. The doublet structure which one might expect as the signature of the $\Lambda$ spin-orbit splitting does not always show up in the spectral distribution since in many cases the matrix elements depend sensitively on binding energies and other wave function effects. As a representative case, we study the influence of the spin-orbit interaction strength on the Auger neutron spectra in more detail for the $1 g^{\Lambda}$ shell. This case is well suited, since the spin-orbit splitting is sufficiently large and the spectrum offers already some complexity. The possible transitions for this configuration together with the corresponding transition rates are shown in table II.

The dependence of the $1 g^{\Lambda}$-shell spectra on variations of the spin-orbit strength of the $\Lambda$-nucleus potential is investigated by changing the relative and the absolute coupling strength of the $\sigma$ and the $\omega$ meson to the $\Lambda$, keeping the overall single particle structure of the $\Lambda$ spectrum fixed. Numerically, this is realized by observing that in our relativistic mean-field theory with scalar and vector self-energies $U_{\sigma}$ and $U_{\omega}$, respectively, the leading order nonrelativistic Schroedinger-type central potential is given by $U_{0}=U_{\omega}-U_{\sigma}$ and the strength of the spin-orbit potential is determined by $U_{l s}=U_{\omega}+U_{\sigma}$. Hence, we can relate spectral effects from variations of the spin-orbit strength by a factor $\mathrm{C}$ to a scaling of the scalar and vector self-energies $U_{\sigma}$ and $U_{\omega}$, respectively, according to

$$
\begin{aligned}
C U_{l s} & =\beta U_{\omega}+\alpha U_{\sigma} \\
D U_{0} & =\beta U_{\omega}-\alpha U_{\sigma} .
\end{aligned}
$$

We preserve the self-consistency between the nuclear mean-field and the underlying baryon-baryon interactions by scaling the $\Lambda \sigma$ and $\Lambda \omega$ vertices, equation (11), by the same factors $\alpha$ and $\beta$, respectively.

Choosing $C$ as our external parameter and using always $D=1$ as a constraint, the spin-orbit splittings of the $\Lambda$ levels can be varied over wide ranges while keeping the changes in the overall structure of the $\Lambda$ single particle spectrum on a minimal level. The $\Lambda$ single particle spectra obtained for $C=0.6,1.3$ (corresponding to $(\alpha, \beta)=(0.6,0.5),(1.3,1,4)$, respectively,) are compared to the results for $C=1$ in figure 6. For $C=0.6$ the spin-orbit splittings are reduced by about a factor of 2 and an increase of about the same size is found for $C=1.3$. These (strong) variations will surely cover the full range of uncertainties about the $\Lambda$ spin-orbit potential. 

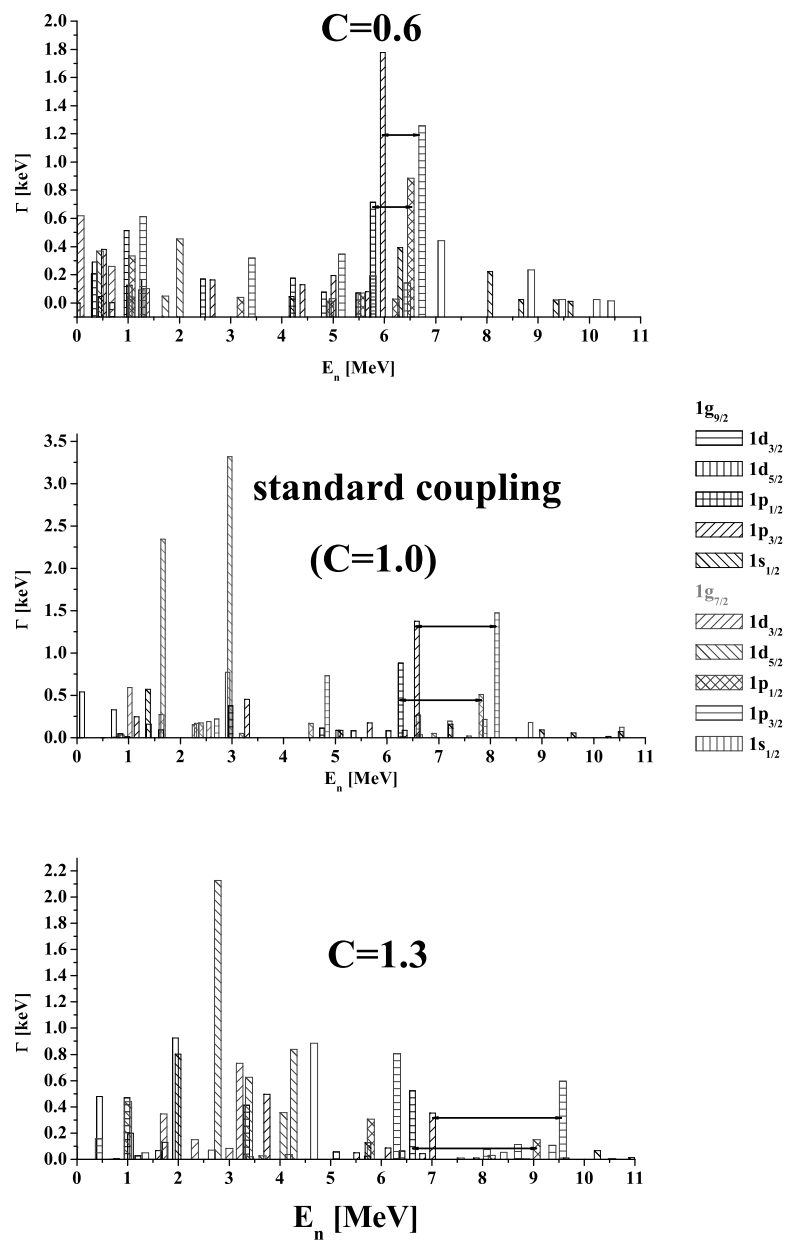

FIG. 6: Comparison of the spectral Auger strength distributions produced by transitions from the $1 g^{\Lambda}$ shell for interactions with different spin-orbit splittings. The Auger transition widths $\Gamma$ are shown as functions of energy $E_{n}$ of the outgoing neutron. The upper and lower arrows indicate transitions from the $1 \mathrm{~g}_{9 / 2,7 / 2}$ to the $1 \mathrm{p}_{3 / 2}$ and $1 \mathrm{p}_{1 / 2}$ final states, respectively.

The spectral distributions of Auger transitions widths $\Gamma$, obtained by putting the initial $\Lambda$ in $1 g_{9 / 2,7 / 2}$ orbits are displayed in figure 6. Compared to the standard case $C=1$ the spectral structures and transition strengths are changed drastically when using $C=0.6$ and $C=1.3$. As an overall feature the calculations show a concentration of strength in a few states for $C=1$ and $C=1.3$ while a more equilibrated distribution is found for $C=0.6$. The apparent pile-up of strength at low neutron energies for the normal and strong coupling cases are related to the variations in the values of overlap matrix elements due to changes in binding energies and single particle wave functions. Analyzing the dynamical content of the response functions by calculating sum rules for various moments of the excitation energy one finds a disappointing small sensitivity on the spin-orbit interaction strength. Hence, it is unlikely that spin-orbit effects will contribute significantly and on an observable level to the total spectral strength.

On the level of individual transitions there are, however, signals for spin-orbit effects visible. In figure 6 the transitions $1 g_{9 / 2,7 / 2}^{\Lambda} \rightarrow 1 p_{3 / 2}^{\Lambda}$ are indicated by the upper arrows while lower arrows denote $1 g_{9 / 2,7 / 2}^{\Lambda} \rightarrow 1 p_{1 / 2}^{\Lambda}$. The length of the arrows corresponds to the $1 g_{9 / 2}^{\Lambda} \leftrightarrow 1 g_{7 / 2}^{\Lambda}$ energy splitting which obviously depends directly on the spinorbit interaction strength. In addition, details of the spectral distributions, e.g. the clustering of strength in certain energy regions, also depends on the overlap of wave functions by which the transition matrix elements and therefore the transition widths $\Gamma$ are determined (see equations (5) and (6)).

It might seem somewhat disadvantageous to start out doing Auger experiments with a system of the complexity of a heavy nucleus like lead. However, aiming at resolving the $\Lambda$ spin-orbit structure in heavy hypernuclei, which is 
still a controversial question as was mentioned in the introduction, one needs to find hypernuclei that provide a core nucleus of very low spin. In the ideal case this should be $\mathrm{J}^{\pi}=0^{+}$in order to eliminate - or at least to suppress effects from $\Lambda$ angular momentum - core nucleus spin interactions leading to an additional splitting superimposed on the pure $\Lambda$ spin-orbit splitting. In this respect the frequently used ${ }^{89} \mathrm{Y}$ and ${ }^{51} \mathrm{~V}$ hypernuclei are very unfavorable cases because the ${ }^{88} \mathrm{Y}$ and ${ }^{50} \mathrm{~V}$ core nuclei carry ground state spins of $4^{-}$and $6^{+}$, respectively! The broadening of the l-orbits observed in these nuclei [1] does not exhibit a proper $\vec{l} \cdot \vec{s}$ systematics which might be related to contribution from residual interactions involving the huge core spins [25]. Hyperisotopes matching the requirements of having a $0^{+}$core ground state and appearing with at least around $10 \%$ natural abundancy would be ${ }_{\Lambda}^{91} \mathrm{Zr}$ and ${ }_{\Lambda}^{119} \mathrm{Sn}$. Lighter isotopes will gradually cease to exhibit Auger de-excitations.

\section{SUMMARY AND CONCLUSIONS}

The Auger-neutron transition rates for the de-excitation of single $\Lambda$ hypernuclei on the example of ${ }_{\Lambda}^{209} \mathrm{~Pb}$ have been calculated in relativistic DDRH theory. The initial state was represented by a ground state ${ }^{208} \mathrm{~Pb}$ core with an attached $\Lambda$ single particle state, the final state as a neutron particle-hole excited ${ }^{208} \mathrm{~Pb}$ where the $\Lambda$ hyperon occupies a lower lying single particle level and the neutron of the particle-hole pair being unbound. Hence the process corresponds to a decay of the initial single $\Lambda$ configuration into a $\Lambda$-neutron-particle-hole configuration.

Due to the fact that the hypernuclear Auger effect appears mainly in heavy and intermediate mass hypernuclei the spectral distribution of the emitted neutrons is extremely complex. For this reason a very detailed reconstruction of each event will have to be done in possible experimental measurements. By focusing on initial states with the $\Lambda$ in the $1 f^{\Lambda}$-orbit our calculations predict a clean doublet structure in the Auger neutron spectrum from which the $1 f^{\Lambda}$ spin-orbit splitting can be directly read off, assuming sufficient energy resolution. For a general $\Lambda$ orbit no definite signature of the spin-orbit splitting will appear due to the huge amount of transitions and the additional broadening of peaks when deeply bound neutrons are involved. The effect of a finite width of the neutron states, increasing with the distance from the Fermi-surface has not been considered in our calculations. For the least bound neutrons it will have almost no effect, but going deeper in binding energy the broadening of the states can no longer be neglected. As described in the previous paragraphs the neutron spectra in which the deeply bound neutrons are also involved are already fairly complex. An additional broadening of the neutron levels will thus wash out most of the spectral structure there. However, in the case of the $1 f^{\Lambda}$-shell de-excitation only the valence neutrons close to the Fermi-level are involved so that the spin-orbit splitting signal is going to survive in realistic spectra.

For the spectroscopy in the intermediate and heavy mass region the hypernuclear Auger effect provides a promising complementary tool to $\gamma$ spectroscopy, although special care must be taken on the choice of transitions and tagging the energy. For the ${ }_{\Lambda}^{209} \mathrm{~Pb}$ hypernucleus the $1 f^{\Lambda}$ shell is such a well suited case. This technique might be the only way to achieve high resolution information on the hypernuclear fine structure in heavy nuclei since for $\gamma$ transitions it will be even harder to assign the detected photons to specific transitions.

\section{ACKNOWLEDGEMENTS}

We would like to thank Amour Margarian for exciting our interest in the hypernuclear Auger effect and for useful discussions on this topic. We would also like to thank Simona Briganti for useful discussions on the general features of the atomic Auger effect. This work was supported by the European Graduate School Gießen-Copenhagen and DFG-contract LE439/4b.

\section{APPENDIX A: MATRIX ELEMENTS}

For the evaluation of the matrix elements the transition form-factors, eq. (7), are evaluated in the spherical basis of Dirac wave functions:

$$
\begin{aligned}
\rho_{12}(q) & =\int d^{3} x \bar{\psi}_{1}(\vec{x}) \hat{\Gamma} \psi_{2}(\vec{x}) e^{i \vec{k} \vec{x}} \\
& =\sum_{\lambda \mu}(-)^{\lambda} Y_{\lambda \mu}^{*}(\hat{q}) \int d \Omega d r r^{2} j_{\lambda}(k r) Y_{\lambda \mu}(\hat{r})\left(\begin{array}{c}
g_{1}(r) \Omega_{j_{1} l_{1} m_{1}}(\theta, \phi) \\
i f_{1}(r) \Omega_{j_{1} \tilde{l}_{1} m_{1}}(\theta, \phi)
\end{array}\right)^{\dagger} \gamma^{0} \hat{\Gamma}\left(\begin{array}{c}
g_{2}(r) \Omega_{j_{2} l_{2} m_{2}}(\theta, \phi) \\
i f_{2}(r) \Omega_{j_{2} \tilde{l}_{2} m_{2}}(\theta, \phi)
\end{array}\right)
\end{aligned}
$$

$\hat{\Gamma}$ is either the four by four unit matrix or $\gamma_{\mu}$, depending whether the scalar or the vector potential is evaluated. $\hat{q}$ and $\hat{r}$ denotes the unit vectors in direction of $\vec{q}$ and $\vec{r}$, respectively. The spinors are the usual total angular momentum 
eigenstates of the Dirac equation with the generalized spin-angle spherical harmonics $\Omega_{j l m}(\theta, \phi)$ obtained by coupling spin and orbital angular momenta [19, 24. The numerical solution of the radial Dirac equation was discussed e.g. in [20]. The orbital angular momenta $l$ and $\tilde{l}$ are determined by $j$ and the parity $\pi$ :

$$
l=\left\{\begin{array}{ll}
j+1 / 2 & \text { for } \pi=(-)^{j+1 / 2} \\
j-1 / 2 & \text { for } \pi=(-)^{j-1 / 2}
\end{array} \quad \tilde{l}= \begin{cases}j-1 / 2 & \text { for } \pi=(-)^{j+1 / 2} \\
j+1 / 2 & \text { for } \pi=(-)^{j-1 / 2}\end{cases}\right.
$$

For the scalar vertex we get then

$$
\begin{aligned}
\rho_{12}^{s}(q)=\sum_{\lambda \mu}(-)^{\lambda} Y_{\lambda \mu}^{*}(\hat{q}) & \left\{\left[\int d r r^{2} g_{1}(r) g_{2}(r) j_{\lambda}(q r)\right]\left[\int d \Omega \Omega_{j_{1} l_{1} m_{1}}^{*} Y_{\lambda \mu} \Omega_{j_{2} l_{2} m_{2}}\right]\right. \\
& \left.-\left[\int d r r^{2} f_{1}(r) f_{2}(r) j_{\lambda}(q r)\right]\left[\int d \Omega \Omega_{j_{1} \tilde{l}_{1} m_{1}}^{*} Y_{\lambda \mu} \Omega_{j_{2} \tilde{l}_{2} m_{2}}\right]\right\}
\end{aligned}
$$

The radial matrix element is evaluated numerically. The angular integral can be performed analytically. By means of the Wigner-Eckhardt theorem [19] it can be expressed by Clebsch-Gordan coefficients and reduced matrix elements

$$
\int d \Omega \Omega_{j l m}^{*} Y_{\lambda \mu} \Omega_{j^{\prime} l^{\prime} m^{\prime}}=\left\langle j l m\left|Y_{\lambda \mu}\right| j^{\prime} l^{\prime} m^{\prime}\right\rangle=(-)^{j-m}\left(\begin{array}{rrr}
j & \lambda & j^{\prime} \\
-m & \mu & m^{\prime}
\end{array}\right)\left\langle l \frac{1}{2} j\left\|Y_{\lambda}\right\| l \frac{1}{2} j^{\prime}\right\rangle
$$

For the vector transition form factor we have to evaluate

$$
\begin{aligned}
\rho_{12}^{\nu}(q)=\sum_{\lambda \mu}(-)^{\lambda} Y_{\lambda \mu}^{*}(\hat{q}) & \left\{\left[\int d r r^{2} g_{1}(r) g_{2}(r) j_{\lambda}(q r)\right]\left[\int d \Omega \Omega_{j_{1} l_{1} m_{1}}^{*} Y_{\lambda \mu} \Omega_{j_{2} l_{2} m_{2}}\right]\right. \\
+ & {\left[\int d r r^{2} f_{1}(r) f_{2}(r) j_{\lambda}(q r)\right]\left[\int d \Omega \Omega_{j_{1} \tilde{l}_{1} m_{1}}^{*} Y_{\lambda \mu} \Omega_{j_{2} \tilde{l}_{2} m_{2}}\right], } \\
\boldsymbol{i} & {\left[\int d r r^{2} g_{1}(r) f_{2}(r) j_{\lambda}(q r)\right]\left[\int d \Omega \Omega_{j_{1} l_{1} m_{1}}^{*} Y_{\lambda \mu} \vec{\sigma} \Omega_{j_{2} \tilde{l}_{2} m_{2}}\right] } \\
+ & {\left.\left[\int d r r^{2} f_{1}(r) g_{2}(r) j_{\lambda}(q r)\right]\left[\int d \Omega \Omega_{j_{1} \tilde{l}_{1} m_{1}}^{*} Y_{\lambda \mu} \vec{\sigma} \Omega_{j_{2} l_{2} m_{2}}\right]\right\}^{\nu} }
\end{aligned}
$$

The $\sigma$ are the usual Pauli matrices. The reduced matrix element for the angular $\rho_{12}^{0}$ matrix element is identical to equation (A4), for the spatial components it is given by

$$
\begin{aligned}
\left\langle j l m\left|Y_{\lambda \mu} \sigma_{M}\right| j^{\prime} l^{\prime} m^{\prime}\right\rangle & =\sum_{I N}\langle\lambda \mu 1 M \mid I N\rangle\left\langle j l m|| Y_{\lambda \mu} \sigma_{1 M}\right]_{I N}\left|j^{\prime} l^{\prime} m^{\prime}\right\rangle \\
& =\sum_{I N}(-)^{j-m}\langle\lambda \mu 1 M \mid I N\rangle\left(\begin{array}{rrr}
j & I & j^{\prime} \\
m & N & m^{\prime}
\end{array}\right)\left\langle l \frac{1}{2} j\left\|\left[Y_{\lambda} \sigma_{1}\right]_{I}\right\| l^{\prime} \frac{1}{2} j^{\prime}\right\rangle \\
& =\sum_{I N}(-)^{j-m}\langle\lambda \mu 1 M \mid I N\rangle\left(\begin{array}{rrr}
j & I & j^{\prime} \\
m & N & m^{\prime}
\end{array}\right)\left\{\begin{array}{ccc}
l & \frac{1}{2} & j \\
l & \frac{1}{2} & j^{\prime} \\
\lambda & 1 & I
\end{array}\right\}\left\langle l\left\|Y_{\lambda}\right\| l^{\prime}\right\rangle\left\langle\frac{1}{2}\|\sigma\| \frac{1}{2}\right\rangle
\end{aligned}
$$

Note that the Pauli matrices $\sigma_{M}, M=0, \pm 1$, are used here in the spherical basis [19]. Explicit expessions for the reduced matrix elements are found e.g. in ref. [19].

[1] H. Hotchi et al., Phys. Rev. C 64, 044302 (2001).

[2] T. Nagae, Nucl.Phys.A 691, 76c (2001) and H. Hotchi, KEK Report 2000-3, April 2000

[3] M. May et al., Phys. Rev. Lett. 78, 4343 (1997)

[4] T. Hasegawa et al., Phys. Rev. C 53, 1210 (1996).

[5] S. Ajimura et al., Nucl. Phys. A 585, 173C (1995).

[6] P. H. Pile et al., Phys. Rev. Lett. 66, 2585 (1991).

[7] M. Hjorth-Jensen, A. Polls, A. Ramos and H. Muther, Nucl. Phys. A 605, 458 (1996) arXiv:nucl-th/9604028.

[8] I. Vidana, A. Polls, A. Ramos and M. Hjorth-Jensen, Nucl. Phys. A 644, 201 (1998) arXiv:nucl-th/9805032.

[9] M. Rufa, H. Stocker, J. A. Maruhn, W. Greiner and P. G. Reinhard, J. Phys. G 13, L143 (1987). 
[10] M. Rufa, J. Schaffner, J. Maruhn, H. Stoecker, W. Greiner and P. G. Reinhard, Phys. Rev. C 42, 2469 (1990).

[11] N. K. Glendenning, D. Von-Eiff, M. Haft, H. Lenske and M. K. Weigel, Phys. Rev. C 48, 889 (1993) [arXiv:nucl-th/9211012.

[12] D. J. Millener, Nucl. Phys. A 691, 93 (2001) arXiv:nucl-th/0103017.

[13] C. Keil and H. Lenske, APS Conf.Proc 603 (Mesons and Light Nuclei 2001), 433 (2001) arXiv:nucl-th/0107068.

[14] C. M. Keil, F. Hofmann and H. Lenske, Phys. Rev. C 61, 064309 (2000) arXiv:nucl-th/9911014.

[15] H. Kohri et al. [AGS-E929 Collaboration], Phys. Rev. C 65, 034607 (2002) arXiv:nucl-ex/0110007.

[16] A. Likar, M. Rosina and B. Povh, Z. Phys. A 324, 35 (1986).

[17] A. Margaryan, L. Tang, S. Majewski, O. Hashimoto, V. Likachev, Auger Neutron Spectroscopy of Nuclear Matter at $C E B A F$, Letter of intent to JLAB PAC 18, LOI-00-101, 2000

[18] see e.g. Particle Data Book, sect. 34, p. 211, Rev. Part. Phys., Europhys.J. C 15 (2000) 1

[19] P.J. Brussaard, P.W.M. Glaudemans, Shell-model applications in nuclear spectroscopy, North-Holland, 1977.

[20] C. Fuchs, H. Lenske and H. H. Wolter, Phys. Rev. C 52, 3043 (1995) arXiv:nucl-th/9507044, H. Lenske and C. Fuchs, Phys.Lett.B 345355 (1995).

[21] F. Hofmann, C. M. Keil and H. Lenske, Phys. Rev. C 64, 034314 (2001) arXiv:nucl-th/0007050.

[22] F. de Jong, H. Lenske, Phys. Rev. C 57, 3099 (1997)

[23] J. Haidenbauer, W. Melnitchouk and J. Speth, arXiv:nucl-th/9805014.

[24] J.D. Bjorken, S.D. Grell, Relativistic Quantum Mechanics, McGraw-Hill, New York, 1964.

[25] This has been found analysing the assumed spin-orbit splittings of [1] within DDRH and phen. RMF. Publication in preparation. 